\title{
On functions preserving rank of matrices
}

\author{
Józef Kalinowski
}




\title{
ON FUNCTIONS PRESERVING RANK OF MATRICES
}

\author{
JÓZEF KALINOWSKI
}

[Received: June 18, 2002]

\begin{abstract}
Aвstract. The paper is motivated by papers of E. Pap [4] and A. Marková [3]. We give a complete characterization of functions preserving ranks of all matrices, without assuming any regularity conditions.

Mathematics Subject Classification: $15 \mathrm{~A} 03$

Keywords: rank of matrices
\end{abstract}

\section{InTRODUCTION}

The notion of g-calculus has been introduced by E. Pap in [4]. A. Marková in [3] observed that a fundamental role in this calculus is played by functions preserving ranks of matrices.

First of all let us introduce

Definition 1. Function $f: \mathbb{R} \longrightarrow \mathbb{R}$ preserves rank of matrices if and only if the rank of a matrix $A=\left[a_{i j}\right]$ is equal to rank of the matrix $f(A):=\left[f\left(a_{i j}\right)\right]$, for every matrix $A$, where $i=1,2 \ldots, n, j=1,2, \ldots, m$.

We proved in [1] that a monotonic and continuous function $f: \mathbb{R} \rightarrow \mathbb{R}$ with $f(0)=0$ preserves ranks of matrices if and only if it is linear, i.e. $f(x)=c \cdot x, x \in \mathbb{R}$, for come constant $c \neq 0$.

In the present paper we show that the characterization holds true even without admitting additional assumptions on function $f$.

\section{Main Results}

It follows immediately from properties of determinants:

Example 1. The linear function $f(x)=c \cdot x$ on $\mathbb{R}$ with a constant $c \neq 0$ is a function preserving rank of matrices.

Without additional assumptions on $f$ (i.e. continuity or others) the following holds true 
Lemma 1. If rank $r(A)=r(f(A))$ for all square matrices of degree less or equal to 3 , then there exists a constant $c \neq 0$ such that $f(x)=c \cdot x$.

Proof. Let $f: \mathbb{R} \longrightarrow \mathbb{R}$ be a function preserving rank of square matrices of degree less or equal to 3 . For matrix $A=[a]$ of degree 1 it follows that $f(a)=0$ if and only if $a=0$. Let $f(1)=c$. From the above it follows that $c \neq 0$. From properties of determinants it follows that $r(A)=r\left(\frac{1}{c} f(A)\right)$. Consider the function

$$
g:=\frac{1}{c} \cdot f \quad \text { on } \quad \mathbb{R},
$$

so that $g(1)=1$. For any square matrix

$$
B=\left[\begin{array}{cc}
1 & b \\
a & a \cdot b
\end{array}\right]
$$

where $a, b \in \mathbb{R}$, we obtain $r(B)=1$. Then also $r(g(B))=1$. Thus

$$
\operatorname{det}\left[\begin{array}{cc}
1 & g(b) \\
g(a) & g(a \cdot b)
\end{array}\right]=0
$$

or $g(a \cdot b)-g(a) \cdot g(b)=0$, i.e. $g$ solves the multiplicative Cauchy equation

$$
g(x \cdot y)=g(x) \cdot g(y) \quad \text { for all } \quad x, y \in \mathbb{R} .
$$

Similarly for any square matrix of the form

$$
C=\left[\begin{array}{ccc}
0 & 1 & a \\
1 & 0 & b \\
1 & 1 & a+b
\end{array}\right]
$$

with arbitrary $a, b \in \mathbb{R}$ the rank $r(C)=2$. Hence also $r(g(C))=2$ and thus

$$
\operatorname{det}\left[\begin{array}{ccc}
0 & 1 & g(a) \\
1 & 0 & g(b) \\
1 & 1 & g(a+b)
\end{array}\right]=0,
$$

i.e. det $C=g(a)+g(b)-g(a+b)=0$. In other words the additive Cauchy functional equation

$$
g(x+y)=g(x)+g(y) \quad \text { for all } \quad x, y \in \mathbb{R}
$$

is fulfilled by $g$. Now, from [2] (Theorem 1, page 356), it follows that the only functions satisfying simultaneously (A) and (M) are $g=0$ or $g=i d$. Since $g(1)=1$, we see that in our case $g(x)=x, x \in \mathbb{R}$. From Definition 1 we obtain that $f(x)=c \cdot x$ for all $x \in \mathbb{R}$.

The following theorem completely describes all functions preserving rank of matrices.

Theorem 1. Function $f: \mathbb{R} \longrightarrow \mathbb{R}$ preserves rank of matrices if and only if $f(x)=$ $c \cdot x$, where $c \neq 0$ is a constant.

Proof. It follows immediately from Lemma 1 and Example 1. 


\section{REFERENCES}

[1] KalinowsKi, J.: On rank equivalence and preserving rank operators, Novi Sad J. Math., 32(1), (2002) (in press).

[2] Kuczma, M.: Functional Equations in a Single Variable, Monografie Mat. 46, Polish Sci. Publ., (PWN), Warszawa, 1968.

[3] Marková, A.: Some remarks on the pseudo-linear algebra, Tatra Mountains Math. Publ., 6, (1995), 123-130.

[4] PAp, E.: g-calculus, Zb. Prir.-Mat. Fak., Univ. Novom Sadu, Ser. Mat. 23, (1993), 145-156.

\section{Author's Address}

\section{Józef Kalinowski:}

Department of Mathematics, Silesian University, 40-007 Katowice, ul. Ban-kowa 14, Poland

E-mail address: kalinows@ux2.math.us.edu.pl 